\title{
An Attractor \\ for a 3D Navier-Stokes Type Equation
}

\author{
F. Gazzola
}

\begin{abstract}
For a modified Navier-Stokes equation existence and uniqueness results are known in the evolution $3 \mathrm{D}$ case: the equation considered is fully nonlinear. We investigate the main properties of the dynamical system defined by the equation: we establish the existence of an attractor, estimate the number of determining modes and prove the global stability of the stationary solution for a "small" external force.
\end{abstract}

Keywords: Navier-Stokes equations, dynamical systems

AMS subject classification: $35 \mathrm{~K} 55,76 \mathrm{D} 05,35 \mathrm{~B} 40$

\section{Setting the equation}

It is well-known that for the Cauchy-Dirichlet problem for the classical 3D Navier-Stokes equations there is a gap between the wider class of functions where existence is known and the smaller classes where uniqueness can be proved. It looks therefore natural to try to modify Navier-Stokes equations in a physically meaningful way in order to obtain existence and uniqueness results. To derive the classical equations a linear relationship between

$$
T=\left\{\tau_{i j}\right\} \quad \text { and } \quad D=\frac{1}{2}\left\{\partial_{i} u_{j}+\partial_{j} u_{i}\right\}
$$

the stress tensor and the deformation velocity tensor, is assumed (here and in the sequel $u_{i}$ represent the components of the velocity vector, while $\partial_{i}=\frac{\partial}{\partial x_{i}}$ and $\partial_{t}=\frac{\partial}{\partial t}$ ), namely the relationship

$$
T=-p I+\mu D
$$

where $p$ denotes the pressure of the fluid, $I$ is the identity $(3 \times 3)$-matrix and $\mu$ is a positive constant representing the coefficient of kinematic viscosity.

A first interesting modification of this relationship is due to Ladyzhenskaya [5] who supposed $T$ to be a continuous function of the components of $D$ satisfying some further conditions and losing its linear feature for large values of the gradient of the velocity. A global unique solvability of the Cauchy-Dirichlet problem is then proved.

Since the Navier-Stokes equations are not of relativistic nature it looks natural to suppose that they break down for high velocities. For this reason, to our belief, it is physically more meaningful to modify relationship. (1) for large values of the velocity

F. Gazzola: Univ. di Torino, Dip. di Sci. Tecnologie Avanzate, 15100 Alessandria, Italy. 
$u$ rather than of its gradient. In a recent work, Prouse [9] remarks that for very high velocities and turbulent flow there is no experimental evidence that the linear relationship (1) continues to hold; he assumes that it holds when the velocity of the fluid is "small" and that it changes in a physically significant way otherwise. More precisely, he assumes that the relationship between the stress tensor and the deformation velocity tensor is given by

$$
\tau_{i j}=-p \delta_{i j}+\frac{1}{2}\left(\partial_{i} \varphi_{j}(u)+\partial_{j} \varphi_{i}(u)\right)
$$

where $\varphi: \mathbb{R}^{3} \rightarrow \mathbb{R}^{3}$ is a function of $u$ whose properties will be given below. Obviously, if $\varphi(u)=\mu u$, relationship (2) reduces to the classical linear law. If we introduce (2) into the general equations of conservation of momentum, the following modified NavierStokes equations for incompressible fluids are obtained:

$$
\begin{aligned}
\partial_{t} u-\Delta \varphi(u)+(u \cdot \nabla) u+\nabla p-\nabla(\nabla \cdot \varphi(u)) & =f \\
\nabla \cdot u & =0 .
\end{aligned}
$$

To establish existence, uniqueness and regularity results for the solution of equations (3) (see Section 2) we assume that $\Omega \subset \mathbb{R}^{3}$ is an open bounded set with boundary $\partial \Omega$ of class $C^{1,1}$ (a function is of class $C^{1,1}$ if its first derivatives are Lipschitz functions). We denote by $L^{p}$ the space of $p$-th power absolutely integrable functions, by $W^{m, p}$ the Sobolev spaces of functions in $L^{p}$ with their first $m$ generalized derivatives in $L^{p}$, by $H^{m}=W^{m, 2}$ the Hilbertian Sobolev spaces, by $H_{0}^{m}$ the $H^{m}$-closure of the space of smooth functions with compact support in $\Omega$, and by $\gamma_{n}$ the normal trace operator. We also need the functional spaces (see [11])

$$
\begin{aligned}
& H=\left\{u \in L^{2}(\Omega): \nabla \cdot u=0 \text { and } \gamma_{n} u=0\right\} \\
& V=\left\{u \in H_{0}^{1}(\Omega): \nabla \cdot u=0\right\}
\end{aligned}
$$

and the dual space $V^{\prime}$ of $V$. It is well-known that $H$ and $V$ are respectively the $L^{2}$ closure and the $H_{0}^{1}$-closure of the space of smooth solenoidal functions with compact support in $\Omega$. Furthermore $H, V$ and $V^{\prime}$ are Hilbert spaces endowed with the scalar products

$$
\begin{aligned}
(u, v)_{H} & =(u, v)_{L^{2}} \\
(u, v)_{V} & =\left((-\Delta)^{1 / 2} u,(-\Delta)^{1 / 2} v\right)_{L^{2}} \\
(u, v)_{V^{\prime}} & =\left(G^{1 / 2} u, G^{1 / 2} v\right)_{L^{2}}
\end{aligned}
$$

where $G: V^{\prime} \rightarrow V$ is the Green operator relative to $-\Delta$.

Even if the results we obtain could be extended to a more general class of functions $\varphi$, we consider the case where $\varphi$ is given by

$$
\varphi(u)=\sigma(|u|) u
$$

since this appears to be a case of particular physical interest (see [9]).

On the function $\sigma$ we make two of the following assumptions: 
(i) $\sigma \in C^{1}[0, \omega), \quad \sigma(\xi) \geq \mu>0, \quad \sigma^{\prime}(\xi) \geq 0$.

(ii) If $\omega<+\infty$, then $\lim _{\xi \rightarrow \omega} \sigma(\xi)=+\infty$.

(ii') If $\omega=+\infty$ and $M>0$ is an arbitrary fixed value, then $\sigma(\xi) \geq \alpha \xi^{s-1}$ when $\xi \geq M(\alpha>0, s \geq 4)$.

(ii") If $\omega=+\infty$ and $M>0$ is an arbitrary fixed value, then $\beta \xi^{s-1} \geq \sigma(\xi) \geq \alpha \xi^{s-1}$ when $\xi \geq M(\beta \geq \alpha>0, s \geq 4)$.

As already noticed, if $\sigma(\xi) \equiv \mu$ when $0 \leq \xi \leq K$, relation (2) becomes the classical linear relationship for $|u| \leq K$. The choice of replacing the linear relationship with (2) looks reasonable if

$$
\varphi(u)=\sigma(|u|) u=(\mu+\bar{\sigma}(|u|)) u \quad \text { with } \bar{\sigma}(\xi) \equiv 0 \text { when } \xi \leq K
$$

and, with the above assumptions on $\sigma,(4)$ can hold.

The main result we prove in this paper states the following.

The dynamical system defined by equations (3) possesses a (weak) attractor $A$ and it is possible to estimate the number of determining modes for the corresponding semigroup $\left\{S_{t}\right\}_{t \geq 0}$ of operators. Furthermore, if the external force $f$ is "small", then the unique stationary solution $u$ of equations (3) is stable and $A \equiv\{u\}$.

The existence of a strong attractor is obtained by Ladyzhenskaya [7] for the equations considered in [5]. The strongest results about attractors for Navier-Stokes equations are obtained in the classical 2D case where the semigroup $\left\{S_{t}\right\}_{t \geq 0}$ has the inclusion properties $S_{t} \in C(H, H)$ and $S_{t} \in L^{\infty}(V, V)$ (see [10: p. 106 - 107] and [1: p. 82]). In our $3 \mathrm{D}$ case we have weaker results and this is due to the full non-linearity of our equation. Indeed the principal part $\Delta \varphi(u)$ is not monotone since we have no information about the sign of $\langle\Delta(\varphi(u)-\varphi(v)), u-v\rangle$ and we cannot infer that $S_{t} \in C(H, H)$. On the other hand we cannot prove the inclusion $S_{t} \in L^{\infty}(V, V)$ since we have no information about the sign of $\langle\Delta \varphi(u), \Delta u\rangle$. For this reason, to obtain estimates, we must choose as test function $G u$ and this leads to a loss of regularity. The classical 2D equations and the modified equations of [5] have a linear and monotone principal part.

In Section 2 we set the Cauchy-Dirichlet problem for equations (3), we recall some known results about existence and uniqueness of a solution and we prove some further regularity properties. In Section 3 we prove some estimates useful in the sequel. In Sections 4 and 5 we establish the properties of the semigroup operators, we prove the existence of an attractor for the dynamical system defined by equations (3) and we estimate the number of determining modes of the semigroup operators $S_{t}(t \geq 0)$. Finally, in Section 6 we suppose the external force $f$ to be constant and "small". We prove that the (unique) stationary solution of equations (3) is stable and that the attractor coincides with it. Further properties of stationary solutions of equations (3) are also given. 


\section{Existence, uniqueness and regularity results}

We consider the following Cauchy-Dirichlet problem for equations $(3)$ in $\Omega \times(0, T)$ :

$$
\begin{aligned}
& u(x, t)=0 \quad \text { if } \quad(x, t) \in \partial \Omega \times(0, T) \\
& u(x, 0)=u_{0}(x) \quad \text { if } \quad x \in \Omega \text {. }
\end{aligned}
$$

We now state two results about existence and uniqueness of a weak solution (in an appropriate sense) of problem (3), (5). The proofs can be found in [9].

Theorem 2.1: Let $\varphi$ be as in (4) and $u_{0}$ as in (5). Assume that $f \in L^{2}\left(0, T ; L^{1}\right) \cap$ $H^{1}\left(0, T ; V^{\prime}\right), u_{0} \in V \cap L^{\infty}$ and that the function $\sigma$ satisfies the assumptions (i), (ii) or (i), (ii ${ }^{\prime}$. Then there exists a unique vector

$$
u \in L^{2}(0, T ; V) \cap L^{s+1}\left(0, T ; L^{s+1}\right) \cap H^{1}(0, T ; H)
$$

such that

$$
\Delta \varphi(u) \in L^{2}\left(0, T ;\left(H^{2}\right)^{\prime}\right)
$$

and

$$
\int_{0}^{T} \int_{\Omega}\left(\left(\partial_{\imath} u+(u \cdot \nabla) u-f\right) \cdot h-\varphi(u) \cdot \Delta h\right) d \Omega d t=0
$$

for all $h \in L^{2}\left(0, T ; H^{2} \cap V\right)$.

Theorem 2.2: Let $\varphi$ be as in (4) and $u_{0}$ as in (5). Assume that $f \in L^{2}\left(0, T ; V^{\prime}\right)$, $u_{0} \in H$ and that the function $\sigma$ satisfies the assumptions (i), (ii"). Then there exists a unique vector

$$
u \in L^{2}(0, T ; V) \cap L^{s+1}\left(0, T ; L^{s+1}\right) \cap W^{1,1+1 / s}\left(0, T ;\left(W^{2, s+1}\right)^{\prime}\right) \cap L^{\infty}(0, T ; H)
$$

such that

$$
\left\langle\partial_{t} u-\Delta \varphi(u)+(u \cdot \nabla) u-f, h\right\rangle=0
$$

for all $h \in L^{2}(0, T ; V) \cap L^{\infty}(0, T ; H) \cap L^{s+1}\left(0, T ; W^{2, s+1}\right)$.

In the sequel we also assume

$$
f \in L^{\infty}\left(\mathbb{R}^{+}, V^{\prime}\right) \quad \text { and } \quad\|f\|_{L^{\infty}\left(\mathbb{R}^{+}, V^{\prime}\right)}=\Phi<+\infty
$$

in order to prove our results, even if this assumption is not necessary to prove Theorem 2.2. Note that assumption (7) implies the inclusion $f \in L_{\text {loc }}^{2}\left(\mathbb{R}^{+}, V^{\prime}\right)$.

For the stationary case we can omit the assumption $s \geq 4$ and consider the more general case where $s \geq 1$; this means that the classical equation can be seen as a particular case of our modified equation. It is proved in [3] that the classical existence and uniqueness results for stationary solutions can be extended to our equation (see also Section 6).

We say that $u \in P_{i}$ if $u$ is the solution of problem (3), (5) relative to the problem of Theorem 2.i $(i=1,2)$. We now state some regularity results of such solutions. The classical result $u \in L^{8 / 3}\left(0, T ; L^{4}\right)$ (see [11: p.297J) holds in our case since $u \in$ $L^{\mathbf{s + 1}}\left(0, T ; L^{\mathbf{s + 1}}\right)$.

From $u \in H^{1}(0, T ; H)$ we infer the following 
Proposition 2.1: If $u \in P_{1}$, then $\partial_{t} u \in L^{2}(0, T ; H)$ and $u \in C(0, T ; H)$.

Proof: It follows from the embedding $H^{1}(0, T ; H) \subset C(0, T ; H)$

Proposition 2.2: If $u \in P_{1}$ or $u \in P_{2}$, then

$$
\begin{aligned}
& u \in L^{\frac{2(\cdot+1)}{\sigma+1+h(1-\sigma)}}\left(0, T ; L^{\frac{o(s+1)}{\sigma+1+h(b-s)}}\right) \\
& \cap L^{\frac{\rho+1}{k}}\left(0, T ; L^{\frac{2(\rho+1)}{\sigma+1+k(1-\sigma)}}\right) \cap L^{\frac{2}{1-r}}\left(0, T ; L^{\frac{6}{2 r+1}}\right)
\end{aligned}
$$

for all $h, k, r \in[0,1]$.

Proof: Since the inclusion $V \subset L^{6}$ the result follows by interpolation between the spaces $L^{2}\left(0, T ; L^{6}\right), L^{\infty}\left(0, T ; L^{2}\right)$ and $L^{s+1}\left(0, T ; L^{s+1}\right)$

Proposition 2.3: If $u \in P_{1}$ or $u \in P_{2}$, then

$$
(u \cdot \nabla) u \in L^{\frac{2}{1+h}}\left(0, T ; L^{\frac{3}{3-h}}\right)
$$

for all $h \in[0,1]$.

Proof: The inclusion $u \in L^{2}(0, T ; V)$ implies the inclusion $\nabla u \in L^{2}\left(0, T ; L^{2}\right)$, but $u \in L^{\infty}(0, T ; H)$, so $(u \cdot \nabla) u \in L^{2}\left(0, T ; L^{1}\right)$. Further, the inclusion $u \in L^{2}(0, T ; V)$ implies the inclusion $u \in L^{2}\left(0, T ; L^{6}\right)$ thus $(u \cdot \nabla) u \in L^{1}\left(0, T ; L^{3 / 2}\right)$. The result now follows by interpolation between $L^{2}\left(0, T ; L^{1}\right)$ and $L^{1}\left(0, T ; L^{3 / 2}\right)$

Proposition 2.4: If $u \in P_{1}$, then

$$
\Delta \varphi(u) \in L^{2}\left(0, T ; L^{1}\right) .
$$

If $f \in L^{1}\left(0, T ; L^{3 / 2}\right)$, then

$$
\Delta \varphi(u) \in L^{\frac{2}{1+h}}\left(0, T ; L^{\frac{3}{3-h}}\right)
$$

for all $h \in[0,1]$.

Proof: Proposition 2.1 yields the inclusion $\partial_{t} u \in L^{2}(0, T ; H)$, thus the inclusion $\partial_{t} u \in L^{2}\left(0, T ; L^{1}\right) \cap L^{1}\left(0, T ; L^{3 / 2}\right)$ is true. The results now follow from Proposition 2.3 and the equality $\Delta \varphi(u)=\partial_{t} u+(u \cdot \nabla) u-f$

Proposition 2.5: If $u \in P_{2}$, then

$$
u \in C\left(0, T ;\left(W^{2, s+1}\right)^{\prime}\right) \quad \text { and } \quad \Delta \varphi(u) \in L^{\cdot+1}+\left(0, T ;\left(W^{2, s+1}\right)^{\prime}\right) .
$$

Proof: The first inclusion is a consequence of the embedding

$$
W^{1,1+1 / s} \cdot\left(0, T ;\left(W^{2, s+1}\right)^{\prime}\right) \subset C\left(0, T ;\left(W^{2, s+1}\right)^{\prime}\right) .
$$

Further, we have the implication

$$
u \in W^{1,1+1 / s}\left(0, T ;\left(W^{2, s+1}\right)^{\prime}\right) \quad \Longrightarrow \quad \partial_{\imath} u \in L^{1+1 / s}\left(0, T ;\left(W^{2, s+1}\right)^{\prime}\right) .
$$

From Proposition 2.3 we infer the inclusion

$$
(u \cdot \nabla) u \in L^{1+1 / s}\left(0, T ;\left(W^{2, s+1}\right)^{\prime}\right)
$$

and the second inclusion then follows from (6) 


\section{Some estimates}

We first state the following technical lemma.

Lemma 3.1: For all elements $u \in L^{5}, v \in L^{5}, w \in H$ and number $\nu>0$ the estimates

$$
\begin{aligned}
|((w \cdot \nabla) u, G w\rangle| & \leq \frac{\nu}{4}\|w\|_{H}^{2}+\frac{128}{\nu^{4}}\|u\|_{5}^{5}\|w\|_{V^{\prime}}^{2} \\
|\langle(w \cdot \nabla) u, G w\rangle+\langle(v \cdot \nabla) w, G w\rangle| & \leq \frac{\nu}{2}\|w\|_{H}^{2}+\frac{128}{\nu^{4}}\left(\|u\|_{5}^{5}+\|v\|_{5}^{5}\right)\|w\|_{V^{\prime}}^{2}
\end{aligned}
$$

are true.

Proof: The first estimate is Lemma 1 in [9]. Indeed with easy calculations one can see that the constant $c_{1}=c_{1}(\nu)$ found there is given by $c_{1}=\frac{128}{\nu^{4}}$. The second estimate follows by applying twice the first one

In what follows we make use of the Poincare inequality

$$
\lambda_{1}^{2}\|u\|_{V^{\prime}}^{2} \leq \lambda_{1}\|u\|_{H}^{2} \leq\|u\|_{V}^{2} \quad \text { for all } u \in V
$$

where $\lambda_{1}>0$ is the first eigenvalue of $-\Delta$ in $\Omega$.

The evolution of a dynamical system is described by a family of operators $\left\{S_{t}\right\}_{t \geq 0}$ that enjoy the semigroup properties: if $u_{0}$ is the initial data (representing the state of the system at time $t=0), u(t)=S_{t} u_{0}$ represents the state of the system at time $t$. See [1: Chapter 2] or [10: Chapter 1] for the basic definitions about dynamical systems.

In order to prove the existence of an attractor for the dynamical system defined by (3), (5) we seek the main properties of the semigroup operators $S_{t}$ acting from $H$ into $H$ relative to problem $P_{2}$. We consider this case since it requires the weaker assumption $u_{0} \in H$.

Theorem 3.1: The semigroup operators $S_{t}$ satisfy the inclusion $S_{t} \in L^{\infty}(H, H)$ for all $t \geq 0$.

Proof: Let us multiply equation (3) by $u(t)$ in $H$ :

$$
\frac{1}{2} \frac{d}{d t}\|u(t)\|_{H}^{2}+\langle\nabla \varphi(u), \nabla u\rangle=\langle f, u\rangle .
$$

Now, use Lemma 2 in [9] to get

$$
\frac{d}{d t}\|u(t)\|_{H}^{2}+2 \mu\|u(t)\|_{V}^{2} \leq \mu\|u(t)\|_{V}^{2}+\frac{1}{\mu}\|f(t)\|_{V}^{2}
$$

that is, by (8),

$$
\frac{d}{d t}\|u(t)\|_{H}^{2}+\mu \lambda_{1}\|u(t)\|_{H}^{2} \leq \frac{1}{\mu}\|f(t)\|_{V^{\prime}}^{2} .
$$

By the Gronwall lemma we have $\left(c=\mu \lambda_{1}\right)$

$$
\|u(t)\|_{H}^{2} \leq\left\|u_{0}\right\|_{H}^{2} e^{-c t}+\frac{\Phi^{2}}{\lambda_{1} \mu^{2}}\left(1-e^{-c t}\right)
$$

where $\Phi$ is given by (7). Finally, we obtain the estimate

which gives the result

$$
\|u\|_{L^{\infty}(\mathbb{R}+; H)}^{2} \leq \rho_{u}=\max \left(\left\|u_{0}\right\|_{H}^{2} ; \frac{\Phi^{2}}{\lambda_{1} \mu^{2}}\right)
$$


Next, we estimate the $L^{5}\left(0, T ; L^{5}\right)$-norm of the velocity $u$.

Proposition 3.1: Let $\alpha$ be as in assumption (ii"), let $u$ be the unique solution of problem (3), (5), let $\rho_{u}$ be as in (10), and let $\delta>\left(\frac{128 \rho_{u}}{\alpha \lambda_{1}}\right)^{1 / 4}$. Then

$$
\int_{t_{1}}^{t_{2}}\|u(t)\|_{5}^{5} d t \leq\left(\alpha-\frac{128 \rho_{u}}{\lambda_{1} \delta^{4}}\right)^{-1}\left(\frac{1}{2}\left\|u\left(t_{1}\right)\right\|_{V^{\prime}}^{2}+\left(\frac{\delta \rho_{u}}{4}+\Phi \sqrt{\frac{\rho_{u}}{\lambda_{1}}}\right)\left(t_{2}-t_{1}\right)\right)
$$

for all $t_{1}, t_{2} \geq 0$.

Proof: From (4.51) in [9] and Lemma 3.1 we infer that, for all $\delta>0$,

$$
\begin{aligned}
\alpha \int_{t_{1}}^{t_{2}}\|u(t)\|_{5}^{5} d t \leq & \frac{1}{2}\left\|u\left(t_{1}\right)\right\|_{V^{\prime}}^{2}+\frac{1}{\sqrt{\lambda_{1}}} \int_{t_{1}}^{t_{2}}\|f(t)\|_{V^{\prime}}\left\|_{u}(t)\right\|_{H} d t \\
& +\int_{t_{1}}^{t_{2}}\left(\frac{\delta}{4}\|u(t)\|_{H}^{2}+\frac{128}{\lambda_{1} \delta^{4}}\|u(t)\|_{5}^{5}\|u(t)\|_{H}^{2}\right) d t \\
\leq & \frac{1}{2}\left\|u\left(t_{1}\right)\right\|_{V^{\prime}}^{2}+\left(\frac{\delta \rho_{u}}{4}+\Phi \sqrt{\frac{\rho_{u}}{\lambda_{1}}}\right)\left(t_{2}-t_{1}\right)+\frac{128 \rho_{u}}{\lambda_{1} \delta^{4}} \int_{t_{1}}^{t_{2}}\|u(t)\|_{5}^{5} d t
\end{aligned}
$$

hence, the result

The next result states that the $L^{2}(0, T ; V)$-norm of the solution $u$ of problem (3), (5) has a growth of at most order $\frac{1}{2}$ when $T \rightarrow+\infty$.

Proposition 3.2: Let $u$ be the unigue solution of problem (3), (5). Then

$$
\mu \int_{t_{1}}^{t_{2}}\|u(t)\|_{V}^{2} d t \leq\left\|u\left(t_{1}\right)\right\|_{H}^{2}+\frac{\Phi^{2}}{\mu}\left(t_{2}-t_{1}\right)
$$

for all $t_{1}, t_{2} \geq 0$.

Proof: From (4.49) in [9] we infer

$$
\mu \int_{i_{1}}^{t_{2}}\|u(t)\|_{V}^{2} d t \leq \frac{1}{2}\left\|u\left(t_{1}\right)\right\|_{H}^{2}+\int_{i_{1}}^{t_{2}}|\langle f(t), u(t)\rangle| d t .
$$

Hence,

$$
\mu \int_{i_{1}}^{t_{2}}\|u(t)\|_{V}^{2} d t \leq\left\|u\left(t_{1}\right)\right\|_{H}^{2}+\frac{1}{\mu} \int_{i_{1}}^{t_{2}}\|f(t)\|_{V}^{2} d t
$$

which, with (7), gives the result 
Finally, we can state that the (unique) solution of problem (3), (5) given by Theorem 2.2 exists for almost every $t \in \mathbb{R}^{+}$.

Proposition 3.3: For all $u_{0} \in H$ the unique solution $u$ of problem (3), (5) satisfies the inclusion

$$
u \in L^{\infty}\left(\mathbb{R}^{+}, H\right) \cap L_{\text {loc }}^{5}\left(\mathbb{R}^{+}, L^{5}\right) \cap L_{\text {loc }}^{2}\left(\mathbb{R}^{+}, V\right) .
$$

Proof: It follows from Theorem 3.1 and the Propositions 3.1 and 3.2

\section{Existence of an attractor}

In this section we prove the existence of an attractor for our dynamical system. We first deal with absorbing sets. From (9) we immediately infer

$$
\limsup _{t \rightarrow+\infty}\|u(t)\|_{H} \leq \frac{\Phi}{\mu \sqrt{\lambda_{1}}}=\rho .
$$

Note that $\rho_{u}=\rho^{2}$ if $\left\|u_{0}\right\|_{H} \leq \rho$. From (11) we get

Proposition 4.1: For all $r \geq \rho$ the balls $B_{H}(r)=\left\{u \in H:\|u\|_{H} \leq r\right\}$ are $H$-absorbing and positively invariant for the semigroup $\left\{S_{t}\right\}_{t \geq 0}$ acting from $H$ into $H$.

We denote by $H_{w}$ the space $H$ endowed with its weak topology and by $H_{V^{\prime}}$ the space $H$ endowed with the strong topology of $V^{\prime}$. $t \geq 0$.

Theorem 4.1: The operators $S_{t}$ satisfy the inclusion $S_{t} \in C\left(H_{V^{\prime}}, H_{V^{\prime}}\right)$ for all

Proof: Let $u$ and $v$ be the solutions of equations (3) corresponding to the initial data $u_{0} \in H$ and $v_{0} \in H$, respectively. We write (6) for $u$ and $v$, subtract the latter from the former, set $w(t)=u(t)-v(t)$ and choose $h=G u$. This leads to

$$
\left\langle w^{\prime}, G w\right\rangle+(\varphi(u)-\varphi(v), w)_{H}=-\langle(w \cdot \nabla) u, G w\rangle-\langle(v \cdot \nabla) w, G w\rangle .
$$

Next use [9: Lemma 3] and Lemma 3.1 (with $\nu=\mu$ ) to get

$$
\frac{d}{d t}\|w(t)\|_{V^{\prime}}^{2}+\mu\|w(t)\|_{H}^{2} \leq K(t)\|w(t)\|_{V^{\prime}}^{2}
$$

where

$$
K(t)=\frac{256}{\mu^{4}}\left(\|u(t)\|_{5}^{5}+\|v(t)\|_{5}^{5}\right) \in L^{1}(0, T)
$$

by Theorem 2.2 . We obtain

$$
\frac{d}{d t}\|w(t)\|_{V^{\prime}}^{2} \leq K(t)\|w(t)\|_{V^{\prime}}^{2} .
$$

Let $h(t)=\frac{1}{2} \int_{0}^{t} K(\tau) d \tau$. Then the Gronwall lemma yields

$$
\|w(t)\|_{v^{\prime}}=\left\|S_{t} u_{0}-S_{t} v_{0}\right\| v^{\prime} \leq e^{h(t)}\left\|u_{0}-v_{0}\right\| v^{\prime}
$$

which gives $S_{t} u_{0} \rightarrow S_{t} v_{0}$ in $H_{V^{\prime}}$ for $u_{0} \rightarrow v_{0}$ in $H_{V^{\prime}}$ 
This result says that if we have a sequence of problems with initial data $u_{0}^{n} \epsilon$ $H(n \in \mathbb{N})$ and $u_{0}^{n} \rightarrow u_{0} \in H$ in $H_{V^{\prime}}$, then $u^{n}(t) \rightarrow u(t)$ in $H_{V^{\prime}}$ for all $t \geq 0$ where $u^{n}$ and $u$ are the solutions of equations (3) corresponding to $u_{0}^{n}$ and $u_{0}$, respectively; $u^{n}(t), u(t) \in H$ by Theorem 2.2. This result will be useful to prove the main property of the semigroup $\left\{S_{t}\right\}_{t \geq 0}$ which is stated in Theorem 4.2 below.

Taking a finer topology for the initial data we obtain

Corollary 4.1: The semigroup operators $S_{t}$ satisfy the inclusions $S_{t} \in C\left(H_{w}, H_{V^{\prime}}\right)$ and $S_{t} \in C\left(H, H_{V}\right)$ for all $t \geq 0$.

We can now prove

Theorem 4.2: The semigroup operators $S_{t}$ satisfy the inclusion $S_{t} \in C\left(H_{w}, H_{w}\right)$ for all $t \geq 0$.

Proof: Let $u_{0}^{n}, u_{0} \in H$ and suppose $u_{0}^{n} \rightarrow u_{0}$ in $H_{w}$. By Corollary 4.1 we have the convergence $S_{t} u_{0}^{n} \rightarrow S_{t} u_{0}$ in $H_{V^{\prime}}\left(S_{t} u_{0} \in H\right)$ and by Theorem 3.1 the sequence $\left\{S_{t} u_{0}^{n}\right\}$ is bounded in $H$. Thus, the sequence $\left\{S_{t} u_{0}^{n}\right\}$ has a $H_{w}$-convergent subsequence (which is the entire sequence) and its $H_{w}$-limit coincides obviously with $S_{t} u_{0}$

Corollary 4.2: The semigroup operators $S_{t}$ satisfy the inclusion $S_{t} \in C\left(H, H_{w}\right)$ for all $t \geq 0$.

Finally, we can prove the existence of an attractor for the dynamical system defined by problem (3), (5).

Theorem 4.3: The dynamical system defined by problem (3), (5) has a $\left(H, H_{w}\right)$ attractor.

Proof: By Theorem 4.2 we have the inclusion $S_{t} \in C\left(H_{w}, H_{w}\right)$. Proposition 4.1 provides the absorbing set $B_{0}=B_{H}(r) \quad(r \geq \rho)$. We have compactness of $B_{0}$ in the topology of $H_{w}$. By using [1: Theorem 2.1/p.121 and Lemma 2.1/p.126] we infer that the dynamical system defined by problem $(3),(5)$ has a $\left(H, H_{w}\right)$-attractor

We denote by $A$ the attractor given by Theorem 4.3 .

\section{The number of determining modes}

In this section we follow a classical procedure (see, for example, [6]) to study the "finite dimension" of the semigroup $\left\{S_{t}\right\}_{t \geq 0}$. We call number of determining modes the smallest integer representing the dimension of $\left\{S_{t}\right\}_{t \geq 0}$ in a sense that will be clear in the sequel. The main problem we meet is that we cannot prove with standard methods (see [10: p. 167]) the backward uniqueness property (i.e. the injectivity of $S_{t}$ ) for our semigroup. Indeed we should prove that, for all $t_{1}$ and $t_{2}$ with $t_{2}>t_{1}$,

$$
\int_{t_{1}}^{t_{2}} \frac{(\varphi(u(t))-\varphi(v(t)), u(t)-v(t))_{H}}{\|u(t)-v(t)\|_{V^{\prime}}^{2}} d t<+\infty
$$

and unfortunately we cannot prove such a result. 
Since the operator $G: L^{2} \rightarrow L^{2}$ is compact and self-adjoint, by the Hilbert-Schmidt theorem we can consider an orthonormal basis $\left\{g_{j}\right\}$ in $H, V$ and $V^{\prime}$ constituted by the eigenfunctions of the operator $-\Delta$ and the corresponding eigenvalues $\left\{\lambda_{j}\right\}$ :

$$
-\Delta g_{j}=\lambda_{j} g_{j} \quad\left(0<\lambda_{1}<\lambda_{2} \leq \ldots \leq \lambda_{j} \rightarrow \infty\right) .
$$

With the scalar products defined in Section 2 we have

$$
\left(g_{j}, g_{k}\right)_{H}=\frac{1}{\sqrt{\lambda_{j} \lambda_{k}}}\left(g_{j}, g_{k}\right)_{V}=\sqrt{\lambda_{j} \lambda_{k}}\left(g_{j}, g_{k}\right)_{V^{\prime}}=\delta_{j k}
$$

and if

$$
v=\sum_{j=1}^{+\infty} \alpha_{j} g_{j} \in H^{s}(\Omega) \quad(s \in \mathbb{R}) \quad \text { then } \quad\|v\|_{H^{*}}^{2}=\sum_{j=1}^{+\infty} \lambda_{j}^{s} \alpha_{j}^{2} .
$$

Denote by $P_{n}$ the (orthogonal) projection from $H$ onto the subspace

$$
H_{n}=\operatorname{span}\left\{g_{1}, \ldots, g_{n}\right\}
$$

Obviously, if $f \in \operatorname{Ker} P_{n}$ (i.e. the first $n$ components of $f$ in $\left\{g_{j}\right\}$ vanish: $f=$ $\left.\sum_{j=n+1}^{+\infty} \alpha_{j} g_{j}\right)$ we have

$$
\|f\|_{V^{\prime}}^{2}=\sum_{j=n+1}^{+\infty} \frac{\alpha_{j}^{2}}{\lambda_{j}} \leq \frac{1}{\lambda_{n+1}} \sum_{j=n+1}^{+\infty} \alpha_{j}^{2}=\frac{1}{\lambda_{n+1}}\|f\|_{H}^{2}
$$

which generalizes'(8).

The next result states that if $u(t)$ and $v(t)$ have the same components on a suitable finite-dimensional subspace $H_{N}$ of $H$, then they have the same $H_{w}$-behaviour as $t \rightarrow$ $+\infty$.

Theorem 5.1: For all $u_{0}, v_{0} \in H$ there exists an integer $N=N\left(\left\|u_{0}\right\|_{H},\left\|v_{0}\right\|_{H}\right)>$ 0 such that the implication

$$
P_{N}\left(S_{t} u_{0}\right)=P_{N}\left(S_{t} v_{0}\right) \text { for all } t \geq 0 \quad \Longrightarrow \quad\left\langle H_{w}\right\rangle \lim _{t \rightarrow+\infty}\left(S_{t} u_{0}-S_{t} v_{0}\right)=0
$$

is true.

Proof: To simplify notations we denote by $c_{i}$ the constants arising in the estimates that follow and we quote their exact value in the appendix. Let $w(t)=u(t)-v(t)$ so that $P_{N}(w(t))=0$ where $N$ will be fixed later. Reasoning like in the proof of Theorem 4.1 yields

$$
\frac{d}{d t}\|w(t)\|_{V^{\prime}}^{2}+\mu\|w(t)\|_{H}^{2} \leq c_{1}\left(\|u(t)\|_{5}^{5}+\|v(t)\|_{5}^{5}\right)\|w(t)\|_{V^{\prime}}^{2}
$$

and, bearing in mind (12),

$$
\frac{d}{d t}\|w(t)\|_{V^{\prime}}^{2} \leq\left(-\mu \lambda_{N+1}+c_{1}\left(\|u(t)\|_{5}^{5}+\|v(t)\|_{5}^{5}\right)\right)\|w(t)\|_{V^{\prime}}^{2} .
$$


Next, divide by $\|w(t)\|_{V}^{2}$, and integrate between 0 and $t(t>0)$ :

$$
\log \frac{\|w(t)\|_{V^{\prime}}^{2}}{\left\|w_{0}\right\|_{V^{\prime}}^{2}} \leq-\mu \lambda_{N+1} t+c_{1} \int_{0}^{t}\left(\|u(\tau)\|_{5}^{5}+\|v(\tau)\|_{5}^{5}\right) d \tau
$$

and by Proposition 3.1 we have (for $\delta$ large enough)

$$
\log \frac{\|w(t)\|_{V^{\prime}}^{2}}{\left\|w_{0}\right\|_{V^{\prime}}^{2}} \leq-\mu \lambda_{N+1} t+c_{2}\left(\left\|u_{0}\right\|_{V^{\prime}}^{2}+\left\|v_{0}\right\|_{V^{\prime}}^{2}+c_{3} t\right) \leq c_{4}+\left(c_{5}-\mu \lambda_{N+1}\right) t .
$$

By the Métivier estimate (see [2] and [8]) we know that there exists a $\bar{\lambda}>0$ such that

$$
\lambda_{n} \sim \bar{\lambda} \cdot n^{2 / 3}
$$

and therefore, we can take $N$ large enough to ensure that $\mu \lambda_{N+1}-c_{5}=K>0$ :

$$
N \sim \Lambda\left(\left\|u_{0}\right\|_{H},\left\|v_{0}\right\|_{H}, \Omega, \Phi\right) \mu^{-15 / 2}+\Lambda^{\prime}
$$

with $\Lambda, \Lambda^{\prime}>0$. With such a choice the Gronwall lemma yields

$$
\|w(t)\|_{V^{\prime}}^{2} \leq c_{6} e^{-K t}\left\|w_{0}\right\|_{V^{\prime}}^{2}
$$

and letting $t \rightarrow+\infty$ we get $\left\langle H_{V^{\prime}}\right) \lim _{t \rightarrow+\infty} w(t)=0$. Finally, by Theorem $3.1, w(t)$ is bounded in $H$ and the result follows

We call complete orbit (or simply orbit) through $u_{0} \in H$ the set of points (when it exists)

$$
\Gamma\left(u_{0}\right)=\bigcup_{t \in \mathbb{R}}\left(S_{t} u_{0}\right)
$$

Sufficient conditions on the external force $f$ can be found to ensure the existence of a complete orbit (see [4]).

Without the backward uniqueness property we cannot prove that the semigroup $\left\{S_{t}\right\}_{t \geq 0}$ can be extended on the attractor $A$ to a continuous group $\left\{S_{t}\right\}_{t \in \mathbb{R}}$ but [1: Proposition 1.3/p. 159] provides the existence of orbits lying entirely on $A$. We can therefore study the behaviour of the group $\left\{S_{t}\right\}_{t \in \mathbb{R}}$ on these orbits. The next result states that this group is in some sense finite-dimensional: for a suitable $N$ the projection of an orbit onto $H_{N}$ determines the orbit itself; the force $f$ must be defined for all $t \in \mathbb{R}$.

Theorem 5.2: Let $f \in L^{\infty}\left(\mathbb{R}, V^{\prime}\right)$ and $\|f\|_{L^{\infty}\left(\mathbb{R}, V^{\prime}\right)}=\Phi$. Then there exists an integer $N>0$ such that, for all $u_{0}, v_{0} \in A$, the implication

$$
P_{N}\left(S_{t} u_{0}\right)=P_{N}\left(S_{t} v_{0}\right) \text { for all } t \in \mathbb{R} \quad \Longrightarrow \quad S_{t} u_{0}=S_{t} v_{0} \text { for all } t \in \mathbb{R}
$$

is true, with $\left\{S_{t}\right\}_{t \in \mathbb{R}}$ being the group acting on $\Gamma\left(u_{0}\right), \Gamma\left(v_{0}\right) \subset A$.

Proof: Reasoning as in the proof of Theorem 5.1 and integrating on $\left[t_{1}, t_{2}\right] \subset \mathbb{R}$ we infer

$$
\log \frac{\left\|w\left(t_{1}\right)\right\|_{V^{\prime}}^{2}}{\left\|w\left(t_{2}\right)\right\|_{V^{\prime}}^{2}} \leq c_{4}+\left(c_{5}-\mu \lambda_{N+1}\right)\left(t_{2}-t_{1}\right)
$$

and therefore

$$
\left\|w\left(t_{2}\right)\right\|_{V^{\prime}}^{2} \leq c_{6} \exp \left(\left(c_{5}-\mu \lambda_{N+1}\right)\left(t_{2}-t_{1}\right)\right)\left\|w\left(t_{1}\right)\right\|_{V^{\prime}}^{2} .
$$

Recall that $\Gamma\left(u_{0}\right), \Gamma\left(v_{0}\right) \subset A$, that is, by (8) and (11), $\|w(t)\| v^{\prime} \leq \frac{2 \Phi}{\mu \lambda_{1}}$ for all $t \in \mathbb{R}$. Choose $N$ big enough so that $\mu \lambda_{N+1}-c_{5}>0$ and let $t_{1} \rightarrow-\infty$ in (16). The result then follows 
The smallest integer $\bar{N}$ for which the implication of Theorem 5.2 holds is called number of determining modes of the group $\left\{S_{t}\right\}_{t \in \mathbb{R}}$ for the orbits lying entirely on the attractor $A$. Using (14) we can estimate $\bar{N}$ as in (15).

\section{Stability of the stationary solution}

In this section we suppose that the external force $f$ is constant $\left(f(t) \equiv f \in V^{\prime},\|f\|_{V^{\prime}}=\right.$ $\Phi)$ and we seek the stability properties of the solutions of the problem

$$
u \in V \cap L^{s+1}: \quad\langle-\Delta \varphi(u)+(u \cdot \nabla) u-f, h\rangle=0 \quad \text { for all } h \in H^{2} \cap V .
$$

Let $\theta=\theta(\Omega)$ be the constant of the embedding $V \subset L^{5}$, i.e.

$$
\|u\|_{5} \leq \theta\|u\|_{V} \quad \text { for all } u \in V
$$

and let $\gamma=\lambda_{1}^{1 / 5} / \theta 2^{9 / 5}$. Then the following existence and uniqueness result holds.

Theorem 6.1: If $\Phi<\gamma \mu^{2}$, then there exists a unique solution of problem (17).

Proof: Existence of a solution of problem (17) is proved in [3] with the usual Galerkin method independently from $\Phi$. If $\Phi<\gamma \mu^{2}$, by Lemma 3.1 with $\nu=\mu$, we have condition (3.22) in [3] which ensures uniqueness of such solution

A similar result holds for the classical Navier-Stokes equations (see [11: Theorem $1.3 /$ p. 167].

For the solution $u$ of problem (17) we have the following estimates (see [3]):

$$
\|u\|_{V} \leq \frac{1}{\mu} \Phi, \quad\|u\|_{H} \leq \frac{1}{\mu \sqrt{\lambda_{1}}} \Phi, \quad\|u\|_{5} \leq \frac{\theta}{\mu} \Phi .
$$

Note that $(u \cdot \nabla) u \in L^{3 / 2} \subset V^{\prime}$ and therefore problem (17) can be formulated in a stronger way, i.e. for all $h \in V$.

From Theorems 5.1 and 5.2 we infer in the next corollaries that the solutions of problem (17) are in some sense finite-dimensional.

Corollary 6.1: For any function $v_{0} \in H$ there exists a number $N=N\left(\left\|v_{0}\right\|_{H}\right) \in$ $\mathbb{N}$ such that if $u$ is a solution of problem (17), then the implication

$$
P_{N}\left(S_{t} v_{0}\right)=P_{N} u \text { for all } t \geq 0 \quad \Longrightarrow \quad\left\langle H_{w}\right\rangle \lim _{t \rightarrow+\infty}\left(S_{t} v_{0}\right)=u
$$

is true.

Corollary 6.2: There exists a number $N>0$ such that the following implications are true.

1) For all $v_{0} \in A$ such that $\Gamma\left(v_{0}\right) \subset A$ and for any solution $u$ of problem (17),

$$
P_{N}\left(S_{t} v_{0}\right)=P_{N} u \text { for all } t \in \mathbb{R} \quad \Longrightarrow \quad S_{t} v_{0} \equiv u
$$


2) For any pair of solutions $u_{1}, u_{2}$ of problem (17),

$$
P_{N u_{1}}=P_{N u_{2}} \quad \Longrightarrow \quad u_{1} \equiv u_{2} \text {. }
$$

A fixed, stationary or equilibrium point is a point $u \in H$ such that $S_{\imath} u=u$ for all $t$. Stationary points $u$ obviously lie on the attractor $A$. We say that $u$ is an asymptotically stable equilibrium if there exists a neighborhood $U$ of $u$ (in $H$ ) such that the implication

$$
v_{0} \in U \Longrightarrow\left\langle H_{w}\right\rangle \lim _{t \rightarrow+\infty}\left(S_{t} v_{0}\right)=u
$$

is true. The following result states that if $\Phi$ is "small", then the unique solution of problem (17) is asymptotically stable.

Lemma 6.1: There exists a $\Psi \in\left(0, \gamma \mu^{2}\right)$ such that if $\Phi<\Psi,\left\|v_{0}\right\|_{H} \leq 2 \rho$ ( $\rho$ as in (11)) and $u$ is the unique solution of problem (17), then.

$$
\left\langle H_{w}\right\rangle \lim _{t \rightarrow+\infty} S_{\imath} v_{0}=u .
$$

Proof: As for Theorem 5.1 we quote the constants $c_{i}$ in the appendix. The constant $\Psi$ will be determined later. For the moment assume only that $\Phi<\gamma \mu^{2}$. Then the uniqueness of $u$ is a consequence of Theorem 6.1. Let $w(t)=v(t)-u$ and choose $h=G w$. Proceding as in Theorem 5.1 we have

$$
\frac{d}{d t}\|w(t)\|_{V^{\prime}}^{2}+\mu\|w(t)\|_{H}^{2} \leq c_{1}\left(\|u\|_{5}^{5}+\|v(t)\|_{5}^{5}\right)\|w(t)\|_{V^{\prime}}^{2}
$$

Next, use (8) and (18) to get

$$
\frac{\frac{d}{d t}\|w(t)\|_{V^{\prime}}^{2}}{\|w(t)\|_{V^{\prime}}^{2}} \leq c_{7}+c_{1}\|v(t)\|_{5}^{5},
$$

then integrate on $[0, t]$ and use Proposition 3.1 with $\delta=\sqrt{32 \rho} / \sqrt[4]{\alpha \lambda_{1}}$ to obtain

Therefore

$$
\log \frac{\|w(t)\|_{V^{\prime}}^{2}}{\left\|w_{0}\right\|_{V^{\prime}}^{2}} \leq c_{8} t+c_{9}
$$

$$
\|w(t)\|_{V^{\prime}}^{2} \leq c_{10}\left\|w_{0}\right\|_{V^{\prime}}^{2} e^{c_{B} t}
$$

and if $\Phi$ is small enough, say $\Phi<\Psi<\gamma \mu^{2}$, then $c_{8}<0$, and letting $t \rightarrow+\infty$ in inequality (19) we have $\left\langle H_{V^{\prime}}\right\rangle \lim _{t \rightarrow+\infty} v(t)=u$. The result then follows by the $H$ boundedness of $v(t)$

Our final result states that in fact, if $\Phi<\Psi$, then the basin of attraction of the stationary solution $u$ is the entire space $H$. This means that $u$ is globally stable and $A \equiv\{u\}$.

Theorem 6.2: Assume that $\Phi<\Psi$ and let $u$ be the unique solution of problem (17). Then, for all $v_{0} \in H$,

$$
\left\langle H_{w}\right\rangle \lim _{t \rightarrow+\infty} S_{t} v_{0}=u
$$

Proof: From (11) we infer that there exists a $\bar{t}>0$ such that $\|v(t)\|_{H} \leq 2 \rho$ for all $t \geq \bar{t}$. Now, consider $w_{0}=v(\bar{t})$ and let $w(t)$ be the unique solution of equations (3) with initial data $w_{0}$ in (5). Therefore

$$
\left\langle H_{w}\right\rangle \lim _{t \rightarrow+\infty} w(t)=\left\langle H_{w}\right\rangle \lim _{t \rightarrow+\infty} v(t) .
$$

Then, by Lemma $6.1,\left\langle H_{w}\right) \lim _{t \rightarrow+\infty} w(t)=u$ and the result follows 


\section{Appendix: exact values of constants}

Exact values of the constants arising in the proof of Theorem 5.1:

$$
R=\max \left(\rho_{u}, \rho_{v}\right) \text { with } \rho_{u} \text { and } \rho_{v} \text { given by (10) }
$$

and

$$
\begin{aligned}
& c_{1}=\frac{256}{\mu^{4}}, \quad c_{2}=\frac{c_{1}}{2}\left(\alpha-\frac{128 R}{\lambda_{1} \delta^{4}}\right)^{-1}, \quad c_{3}=\delta R+4 \Phi \sqrt{\frac{R}{\lambda_{1}}} \\
& c_{4}=c_{2}\left(\left\|u_{0}\right\|_{V^{\prime}}^{2}+\left\|v_{0}\right\|_{V^{\prime}}^{2}\right), \quad c_{5}=c_{2} c_{3} \sim c \mu^{-4}, \quad c_{6}=e^{c_{4}} .
\end{aligned}
$$

Exact values of the constants arising in the proof of Lemma 6.1:

$$
\begin{array}{cl}
c_{7}=c_{1}\left(\frac{\theta \Phi}{\mu}\right)^{5}-\mu \lambda_{1}, & c_{8}=c_{7}+c_{1} \frac{2}{\alpha}\left(\frac{\sqrt{32 \rho}}{\sqrt[4]{\alpha \lambda_{1}}} \rho^{2}+\frac{2 \Phi \rho}{\sqrt{\lambda_{1}}}\right) \\
c_{9}=\frac{4 c_{1} \rho^{2}}{\alpha \lambda_{1}}, & c_{10}=e^{c_{9}}, \quad \rho_{u}=4 \rho^{2} .
\end{array}
$$

\section{References}

[1] Babin, A. V. and M. I. Visik: Attractors of Evolution Equations. Amsterdam et al: North-Holland Publ. Comp. 1992.

[2] Foias, C. and R. Temam: Some analytic and geometric properties of the solutions of the evolution Navier-Stokes equations. J. Math. Pures Appl. 58 (1979), 339 - 368.

[3] Gazzola, F.: Existence and uniqueness results for a generalized stationary Navier-Stokes equation. Rend. Ist. Lomb. Sci. Lett. (Sez. A) 126 (1992), 181 - 199.

[4] Iannelli, A.: Bounded and almost periodic solutions of a Navier-Stokes type equation. Rend. Acc. Naz. XL 15 (1991)4, $71 \cdot 91$.

[5] Ladyzhenskaya, O.: On some modifications of the Navier-Stokes equations for large gradients of velocity. Zap. Nauchn. Sem. LOMI V 7 (1968), 126 - 154.

[6] Ladyzhenskaya, O.: Attractors for Semigroups and Evolution Equations. Lezioni Lincee (Roma 1988). Cambridge: Univ. Press 1991.

[7] Ladyzhenskaya, O.: Attractors for the modifications of the three-dimensional NavierStokes equations. Philos. Trans. Roy. Soc. London (Ser. A) 346 (1994), 173-190.

[8] Metivier, G.: Valeurs propres d'opérateurs définis par la restriction de systèmes variationnels à des sous-espaces. J. Math. Pures Appl. 57 (1978), 133 - 156.

[9] Prouse, G.: On a Navier-Stokes type equation. In: Nonlinear analysis: a tribute in honour of Giovanni Prodi (eds.: A. Ambrosetti and A. Marino). Quaderni Sc. Norm. Sup. Pisa 1991, pp. 289 - 305.

[10] Temam, R.: Infinite-Dimensional Dynamical Systems in Mechanics and Physics (Applied Mathematical Sciences: Vol. 68). New York et al: Springer - Verlag 1988.

[11] Temam, R.: Navier-Stokes Equations. Theory and Numerical Analysis (Studies in Mathematics and its Applications: Vol. 2). 3rd. ed. Amsterdam et al: North - Holland Publ. Comp. 1984. 